\title{
S1 domain of the porcine epidemic diarrhea virus spike protein as a vaccine antigen
}

Niraj Makadiya, Robert Brownlie, Jan van den Hurk, Nathalie Berube, Brenda Allan, Volker Gerdts and Alexander Zakhartchouk ${ }^{*}$

\begin{abstract}
Background: Porcine epidemic diarrhea virus (PEDV) is a highly contagious virus infecting pigs of all ages with high morbidity and mortality among newborn piglets. Currently, there is no effective vaccine available to protect the pigs from PEDV. The N-terminal subunit of spike protein (S1) is responsible for virus binding to the cellular receptor and contains a number of neutralizing antibody epitopes. Thus, we expressed and produced recombinant S1 protein to protect newborn piglets by immunization of sows.

Methods: Affinity tagged PEDV S1 protein was expressed in a secretory form in yeast, insect and mammalian cells to identify the most suitable production system. Purified recombinant protein was analysed by SDS-PAGE, Western blot and deglycosylation assay. A pregnant sow was intramuscularly immunized three times with adjuvanted recombinant protein prior to farrowing. PEDV-specific immune responses in sera and colostrum of the sow and piglets were assayed by ELISA and virus neutralization assays. Piglets were challenged orally with PEDV, and clinical parameters were monitored for 6 days post-challenge.

Results and conclusion: Of three eukaryotic expression systems tested (yeast, insect-cell, and mammalian), expression by HEK-293 T cells gave the highest yield of protein that was N-glycosylated and was the most appropriate candidate for vaccination. Administration of the subunit vaccine in a sow resulted in induction of S1-specific $\lg G$ and IgA that were passively transferred to the suckling piglets. Also, high virus neutralization titres were observed in the serum of the vaccinated sow and its piglets. After PEDV challenge, piglets born to the vaccinated sow exhibited less severe signs of disease and significantly lower mortality compared to the piglets of a control sow. However, there were no significant differences in diarrhea, body weight and virus shedding. Thus, vaccination with S1 subunit vaccine failed to provide complete protection to suckling piglets after challenge exposure, and further improvements are needed for the development of a subunit vaccine that fully protects against PEDV infection.
\end{abstract}

Keywords: PEDV, S1, Subunit vaccine, Lactogenic immunity

\section{Background}

Porcine epidemic diarrhea virus (PEDV) is an enveloped, positive-stranded RNA virus which readily infects pigs, resulting in highly contagious porcine epidemic diarrhea [1]. PEDV belongs to family Coronaviridae, subfamily Coronavirinae and genus Alphacoronavirus [2]. Some

\footnotetext{
* Correspondence: alex.zak@usask.ca

Vaccine and Infectious Disease Organization - International Vaccine Center (VIDO-InterVac), University of Saskatchewan, 120 Veterinary Road, Saskatoon, SK S7N 5E3, Canada
}

viruses of the Coronaviridae family cause severe disease in humans such as severe acute respiratory syndrome coronavirus (SARS-CoV) and Middle East respiratory syndrome coronavirus (MERS-CoV) [3, 4]. Coronaviruses of veterinary significance include avian infectious bronchitis virus infecting chickens, transmissible gastroenteritis virus (TGEV) infecting pigs, bovine coronavirus, feline coronaviruses, canine coronavirus and turkey coronavirus [5]. 
Porcine epidemic diarrhea (PED) was first observed in Europe in the early 1970s, and PEDV was first isolated in Belgium in 1978 [6]. Subsequently, PED has become an endemic disease in Asian pig farming countries. Severe PED outbreaks were reported in China in 2010-2012 [7, 8]. From April 2013 to the present, major PEDV outbreaks have been reported in the USA [9], Canada [10], Taiwan [11] and Europian countries $[12,13]$. The PED is characterized by the presence of watery diarrhea in the infected piglets in first few weeks of their life, dehydration, vomiting and anorexia resulting in high morbidity and mortality [14]. PEDV infection of older pigs results in considerably lower morbidity and mortality. The symptoms of the disease are similar to transmissible gastroenteritis of pigs and hence only laboratory tests can aid in differencial diagnosis [15]. Although, some efforts have been made to create the vaccine against PEDV with varied success, no effective vaccine is available in the market to protect the newborn piglets $[14,15]$.

The size of PEDV genomic RNA is about $28 \mathrm{~kb}$, and contains seven open reading frames (ORFs) encoding viral proteins: 1A, 1B, spike (S), ORF3, envelope (E), membrane $(\mathrm{M})$ and nucleocapsid $(\mathrm{N})$. The $\mathrm{S}$ protein is present at the outer surface of the virion and is 1386 amino acid long [16]. The spike protein of coronaviruses forms trimers and plays an important role in the virus attachment and in virus-cell membrane fusion [17]. Porcine aminopeptidase $\mathrm{N}$ has been demonstrated to be a functional receptor for the PEDV coronavirus [18]. The S protein of PEDV is a class I membrane glycoprotein consisting of two subunits: the N-terminal S1 and the C-terminal S2. Cleavage of spike protein into S1 and S2 is an essential event in the cellular entry for wild-type PEDV virus but not for cell culture adapted PEDV [19]. Proteolytic cleavage of spike protein in PEDV needs trypsin $[19,20]$. Several neutralizing epitopes have been identified on the $S$ protein sequence [21-23], and the recombinant $S 1$ protein was previously shown to have protective activity in piglets [24].

\section{Results and discussion}

\section{Expression of $\mathrm{S} 1$ in yeast cells}

Initial attempts to express the S1 protein in the bacterial cells were not successful (data not shown), which may be due to problems in processing of the S1 protein in prokaryotic cells. Therefore, we used PichiaPink (Pichia pastoris) yeast cells to express S1 from a synthetic S1 gene codon optimized for yeast and containing a C-terminal histidinetag to aid purification. Initially, the time course was performed for the expression of the S1 protein in the yeast cells over the period of 4 days. Western blot analysis of the cell culture medium of transformed yeast cells resulted in the detection of a specific $35-40 \mathrm{kDa}$ band when probed with anti-his antibody (Fig. 1a). The observed protein molecular weight was less than the expected $80.9 \mathrm{kDa}$ molecular weight of un-glycosylated S1. This may be due to the cleavage of the protein by yeast protease. The purified protein was detected in SDS-PAGE as a smearing band in a range of $40-70 \mathrm{kDa}$ (Fig. 1b). The band pattern may be the result of glycosylation of multiple sites in the $S 1$ protein by the yeast cells. The yield of the purified protein from one liter of yeast culture was found to be $180 \mu \mathrm{g}$.

\section{Expression of S1 by recombinant baculovirus}

To increase the PEDV S1 protein yield and to achieve a full-length S1 protein expression, a baculovirus expression system was employed. To this end, histidine-tagged PEDV S1 gene was cloned into a BACMID containing the genome of baculovirus, transfected into Sf9 cells and the recombinant baculovirus was recovered. Small scale PEDV S1 protein expression in the recombinant baculovirus infected cells was analysed by Western blotting using the anti-his antibody at days 1 to 7 post-infection (Fig. 2a). The S1 protein was detected as a distinct $100 \mathrm{kDa}$ band and band intensity increased on each subsequent day. Size and integrity of affinity purified S1 from the cell culture medium of baculovirus infected cells was found to be of the same size (Fig. 2b) as detected earlier (Fig. 2a) with the yield of $3.9 \mathrm{mg} / \mathrm{L}$.

\section{Expression of $\mathrm{S} 1$ in mammalian cells}

Mammalian expression of S1 utilizing a human CMV promoter within an episomal vector was also investigated. In preliminary experiments, expression of S1 in which the native signal peptide was replaced with that of tissue plaminogen activator (TPA) was compared with expression of S1 containing native signal peptide. Transformed HEK-293 T cells were grown for $24 \mathrm{~h}$ in serum free medium, and the supernatant was then analysed by Western blotting for expression of histidine-tagged protein. The S1 protein was detected as a single $130 \mathrm{kDa}$ band for both cells expressing S1 with native signal peptide and cells expressing S1 with TPA (Fig. 3a). However, the presence of TPA substantially enhanced expression of S1. Therefore, S1 with TPA was subsequently used for production and purification of the vaccine antigen. Affinity purified S1 protein was analysed on SDS-PAGE by Coomassie blue staining and found to be of the expected size (Fig. 3b). The yield of the S1 protein from HEK $293 \mathrm{~T}$ cell culture medium was found to be $30 \mathrm{mg} / \mathrm{L}$, which was 10 and 100-fold higher than the yields obtained from insect cells or yeast cells respectively.

\section{Glycosylation profile of the purified S1 protein}

As there are multiple glycosylation sites present in the spike proteins of other coronaviruses, the purified PEDV S1 protein was analysed for glycosylation. Sensitivity to 

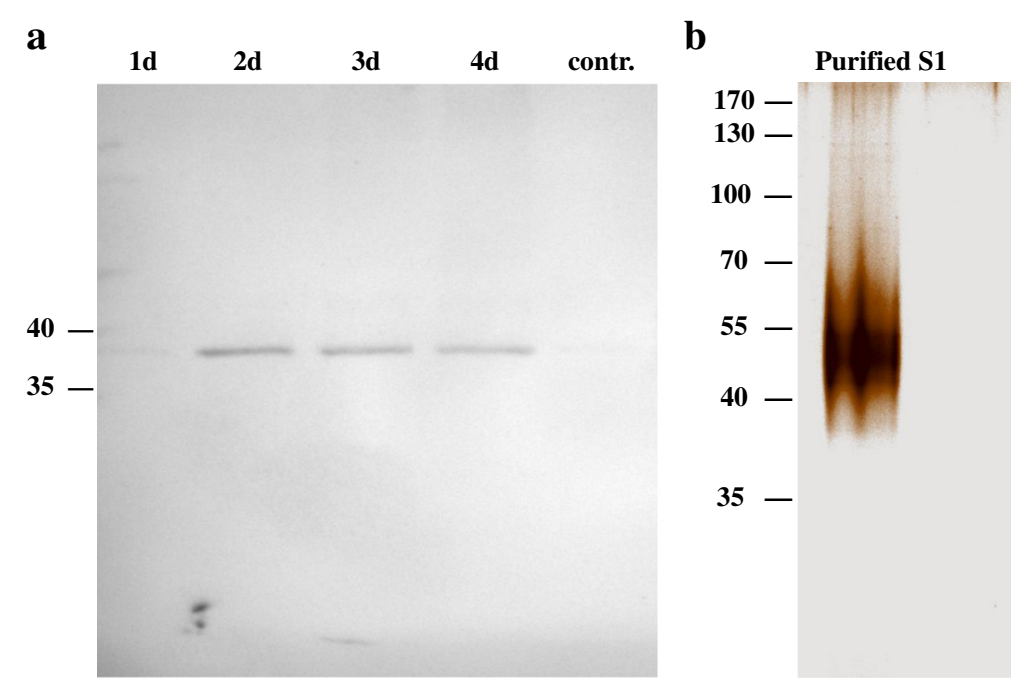

Fig. 1 Expression of the recombinant PEDV S1 protein in PichiaPink Pichia pastoris. a A small scale S1 protein expression experiment was performed, and samples of culture medium were collected on indicated days post-induction and detected by Western blotting using anti-his antibody. b A large scale S1 protein purification was performed from the yeast cell supernatant at day 4 post-induction, and the purified protein was anyalzed by staining of SDS-PAGE by silver nitrate. Medium from the un-induced cells served as a negative control. Numbers on the left indicate the protein molecular marker size in $\mathrm{kDa}$

glycosidases PNGase F (removes N-linked glycans) and $O$-glycosidase (removes O-linked glycans) was used to determine the nature of glycosylation of the recombinant protein.

PNGase $\mathrm{F}$, but not $\mathrm{O}$-glycosidase, increased the electrophoretic mobility of purified recombinant S1 produced by both HEK-293 T or baculovirus-infected cells (Fig. 4) suggesting that the recombinant protein is $\mathrm{N}$ - but not $\mathrm{O}$-glycosylated when produced by either mammalian or insect cells.

\section{Humoral immune responses in sows}

To determine the immunogenicity of the S1-based subunit vaccine, a pregnant sow was immunized
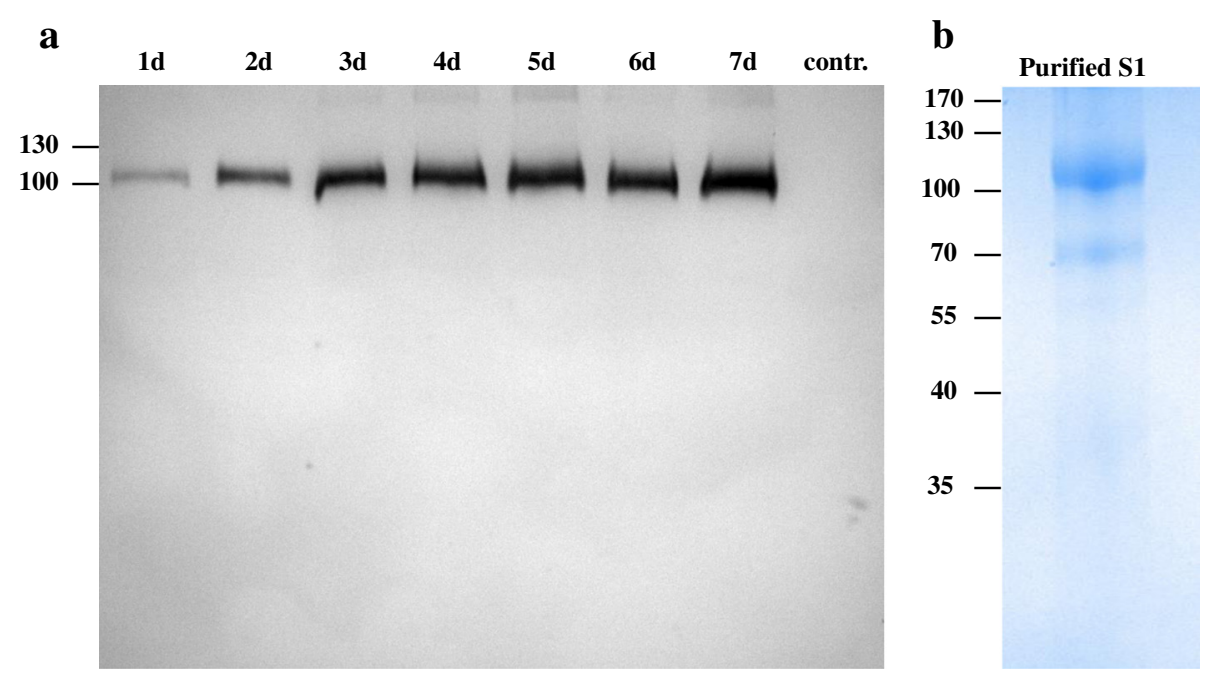

Fig. 2 Expression of the recombinant PEDV S1 protein in insect Sf9 cells. a A small scale S1 protein expression experiment was performed by infecting Sf9 cells with recombinant baculovirus expressing PEDV S1 gene. Cell culture media were collected on indicated days post-infection and analysed by Western blotting using anti-his antibody. b Large scale S1 protein purification was performed on cell culture medium of the cells infected with recombinant baculovirus at day 7 post-infection, and the purified protein was anyalzed by SDS-PAGE and stained by Coomassie Blue. Medium from un-infected cells served as a negative control. Numbers on the left indicate the protein molecular marker size in $\mathrm{kDa}$ 


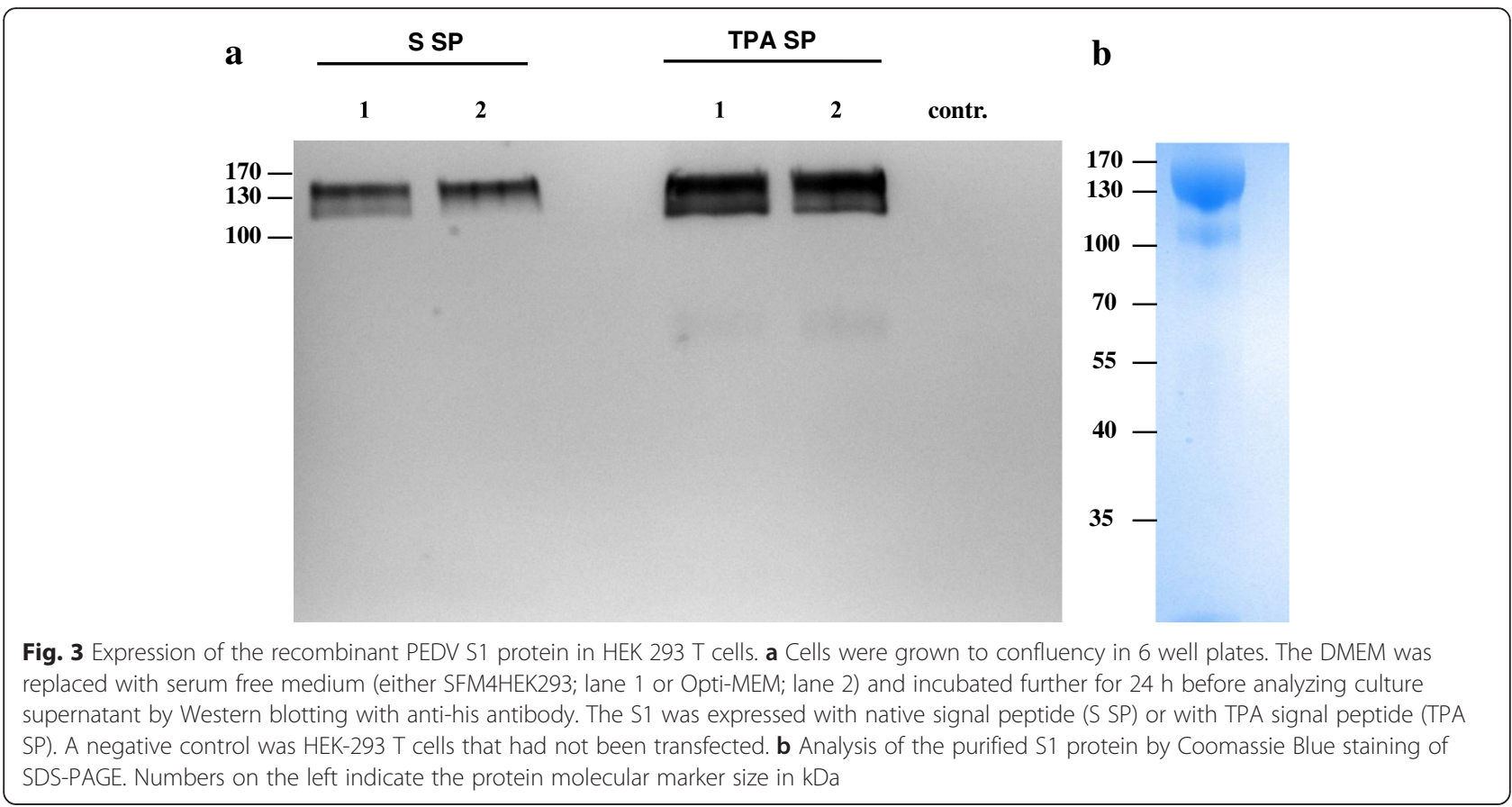

intramuscularly with adjuvanted S1 recombinat protein produced from HEK $293 \mathrm{~T}$ cells. The humoral immune responses elicited by the subunit vaccine were examined by ELISA and serum neutralization assay. The vaccinated sow showed an increase in S1- specific serum IgG titer after the first, second and third vaccination (Fig. 5a) whereas no increase was noticed in the control sow. Furthermore, in contrast to the control sow, vaccinated sow demonstrated detectable serum neutralizing-antibody titers at day 11

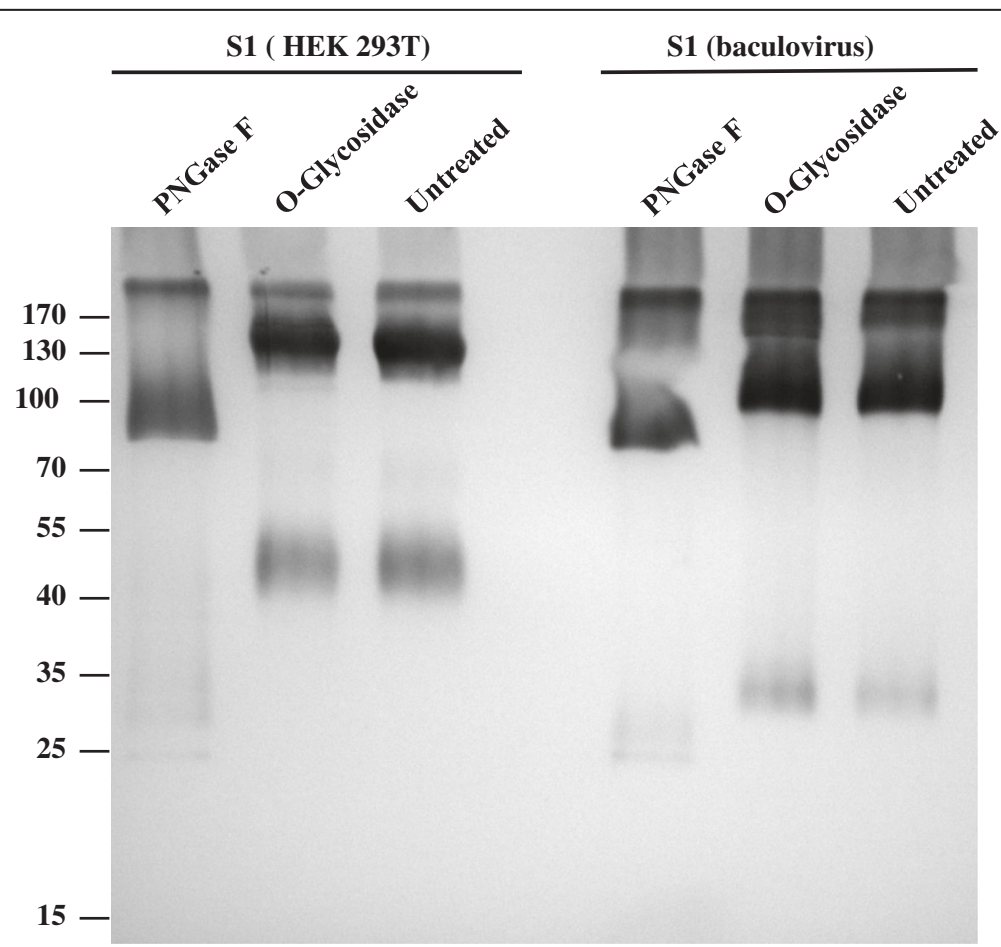

Fig. 4 Deglycosylation analysis of the purified recombinant S1 proteins. S1 proteins purified from cell culture media of HEK 293 T cells or baculovirus infected Sf9 cells were treated with deglycosylation enzymes PNGase F or O-Glycosidase or left un-treated. Proteins were resolved on $10 \%$ SDS-PAGE and analysed by Western blotting using anti-his antibody. Numbers on the left indicate the protein molecular marker size in kDa 


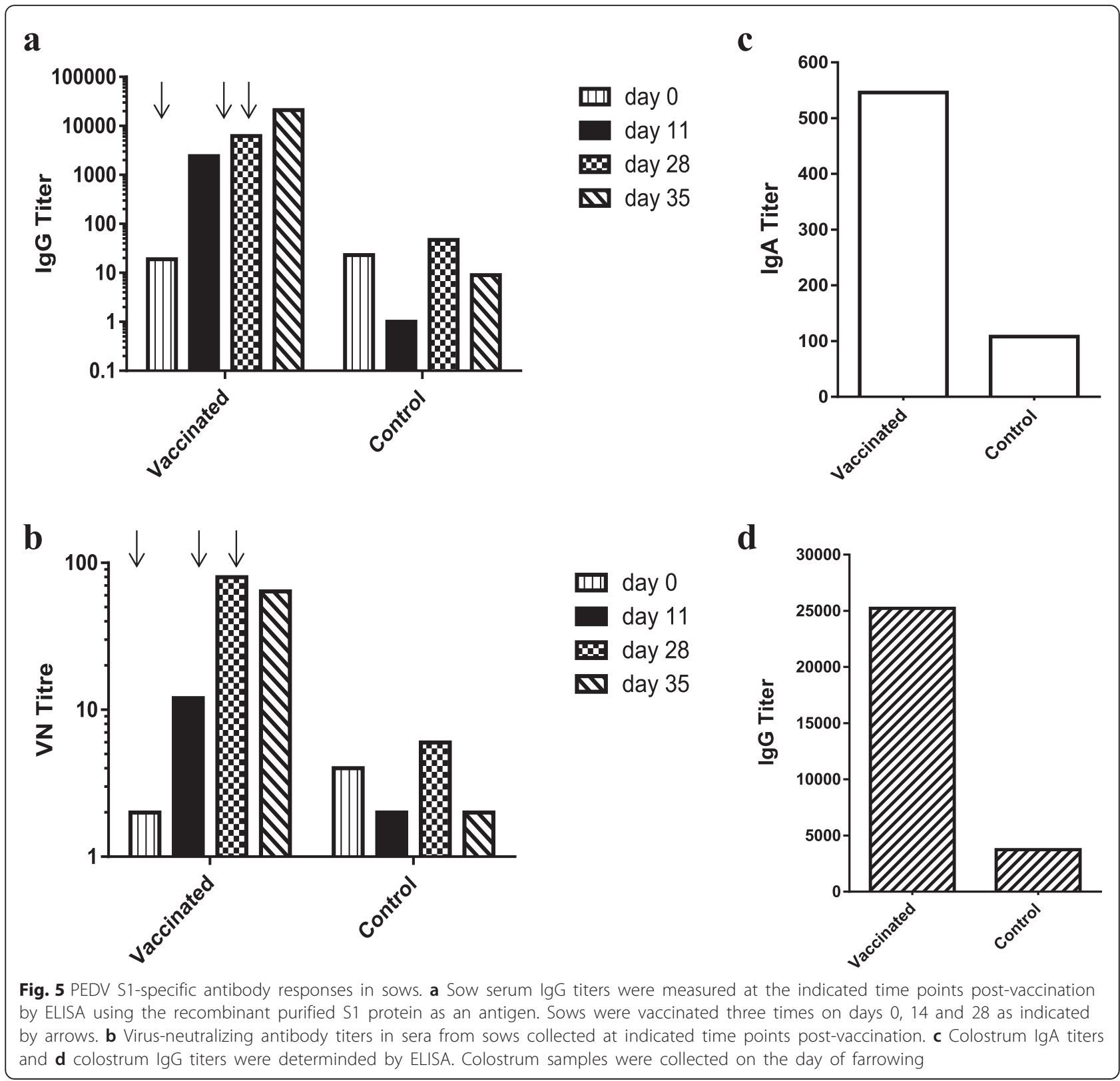

post-vaccination, and by 28 days the titers had increased 100-fold (Fig. 5b). In contrast to ELISA titers (Fig. 5a), virus neutralizing titers did not increase after the third immunization of sow by day 35 (Fig. 5b).

Piglets that regularly suckle the immune mother receive colostrum/milk antibody, a process that cofers passive immunity to the piglets. Therefore, we tested colostrum samples collected from the vaccinated and control sows on the day of farrowing for the presense of PEDV S1-specific IgA and IgG antibody titres. The IgA and IgG titers were higher in colostrum of the vaccinated sow compared to the control sow (Fig. 5c and d). The IgG titer levels were 5-fold higher than IgA titer levels, but it is not surprising since IgG is the major isotype in sow colostrum whereas IgA predominates in milk [25].

\section{Humoral immune responses in piglets}

Sera of piglets, collected on day 4 after birth, had high titres of S1-specific IgG in the litter of a vaccinated sow, while no titres were found in the litter of a control sow (Fig. 6a). These data indicate that passive transfer of S1-specific IgG antibodies occurred between the vaccinated pregnant sow and the offspring via colostrum/milk. In addition, these specific antibodies also conferred virus neutralization (Fig. 6b). 

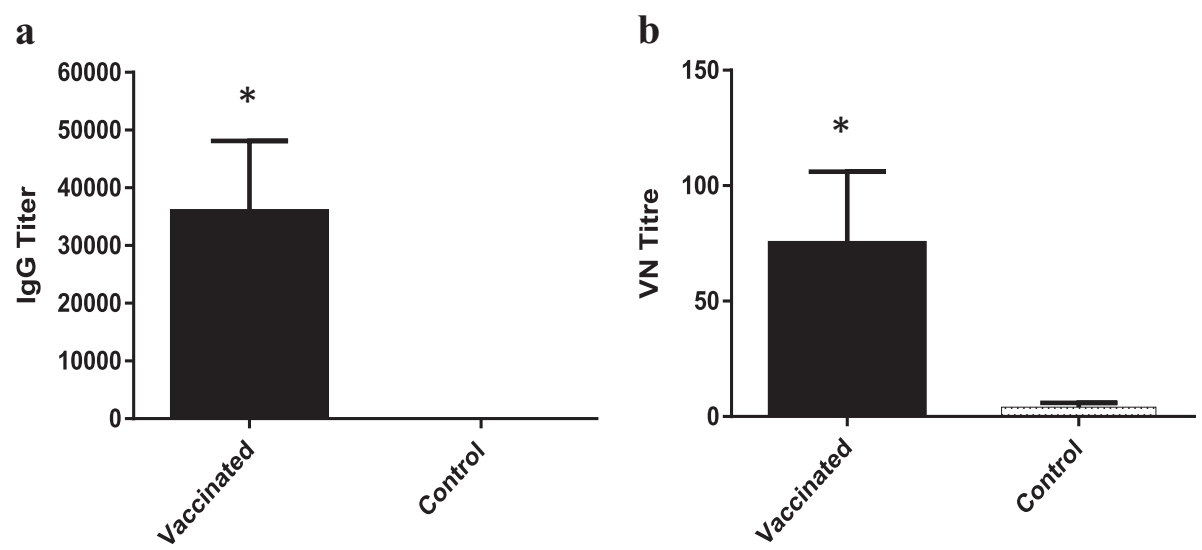

Fig. 6 PEDV S1-specific antibody titers in piglets. a Piglet serum IgG titers were measured on the day of PEDV challenge by ELISA. The values represent the mean $\lg G$ titers of litters from a vaccinated sow $(n=8)$ and litters $(n=7)$ of an unvaccinated control sow. Error bars represent the standard deviation. $\mathbf{b}$ Virus-neutralizing antibody titers in sera from piglets at the day of PEDV challenge. The error bars represent the standard deviation of the mean. ${ }^{*}$ means significantly different results $(P<0.001)$

\section{Virus shedding in challenged piglets}

Virus shedding in feces of piglets collected at different time points post challenge was assessed by a PEDV $\mathrm{N}$ gene-based real-time RT-PCR, and the cycle threshold $\left(C_{T}\right)$ values are shown in Fig. 7. No differences were observed in PEDV shedding between groups of piglets of vaccinated and control sow.

\section{Clinical observations}

There were no adverse reactions noticed at the injection site or overall health of sow post-vaccination. Each day post-challenge, the piglets were evaluated by animal services veterinarian for the clinical scores. The clinical score was assigned as 0 (healthy) to 4 (dead or moribund) for each piglet based on parameters such as animal demeanor, degree of depression and willingness to nurse. The average clinical scores for the vaccinated

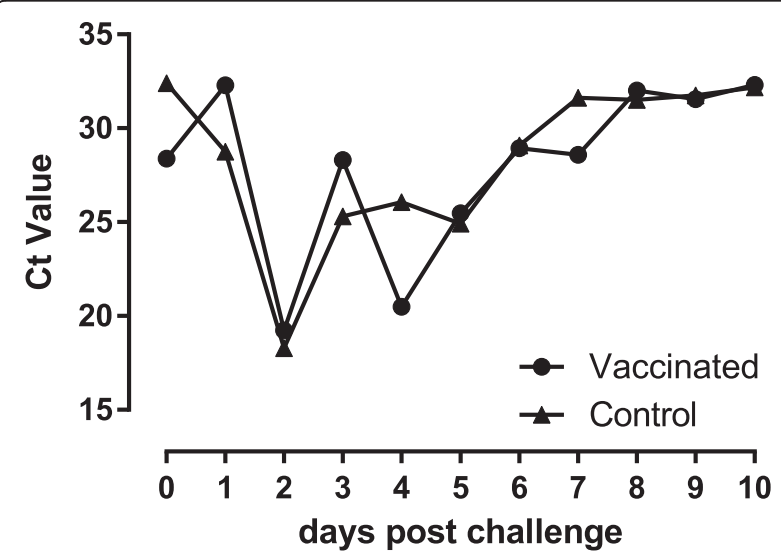

Fig. 7 Virus shedding in piglets. PEDV viral RNA was extracted from rectal swabs of challenged piglets and assessed by real-time reverse transcription PCR sow's piglets was close to zero, whereas the control sow's piglets had average scores reaching on day 2 and 3 postchallenge (Fig. 8a). Similarly, fecal scores were recorded from 0 (normal pasty faces) to 2 (watery diarrhea) for all the piglets each day post-challenge. Diarrhea increased until day 4 post-challenge and then started to reduce for both groups of piglets (Fig. 8b).

The weight of each piglet was monitored every day post-farrowing for 10 days, and the analysis of the average weight of piglets showed no appreciable differences between two groups of piglets (Fig. 8c).

Survival of the piglets was monitored for 10 days after challenge. At 6 days post-challenge, 7 out of 8 piglets $(87.5 \%)$ of vaccinated sow survived whereas only 3 out of 7 piglets (42.9\%) of control sow survived the challenge (Fig. 8d). Statistically significant $(P=0.0002)$ better survival rate in the litter of vaccinated sow can be explained by the fact that these piglets were less depressed and showed more willingness to nurse than piglets of a control sow. However, more experiments with large number of sows are needed to confirm these data.

In summary, our vaccine had negligible effect in either preventing diarrhea or preventing PEDV-mediated weight loss, but did partially protect piglets in terms of severity of clinical disease and significantly reduced mortality. Future work to improve the protective efficacy of the subunit vaccine for PEDV may include testing new adjuvants. For instance, in the recently published report, oil-in-water adjuvant was used to formulate recombinant S1 protein [24]. Another approach is use of oral immunization instead of intramuscular (IM) route. A field study demonstrated that orally vaccinated sows with live attenuated PEDV vaccine exhibited higher IgA and virus neutralizing antibody levels in the colostrum or sera compared to those of the counterparts 


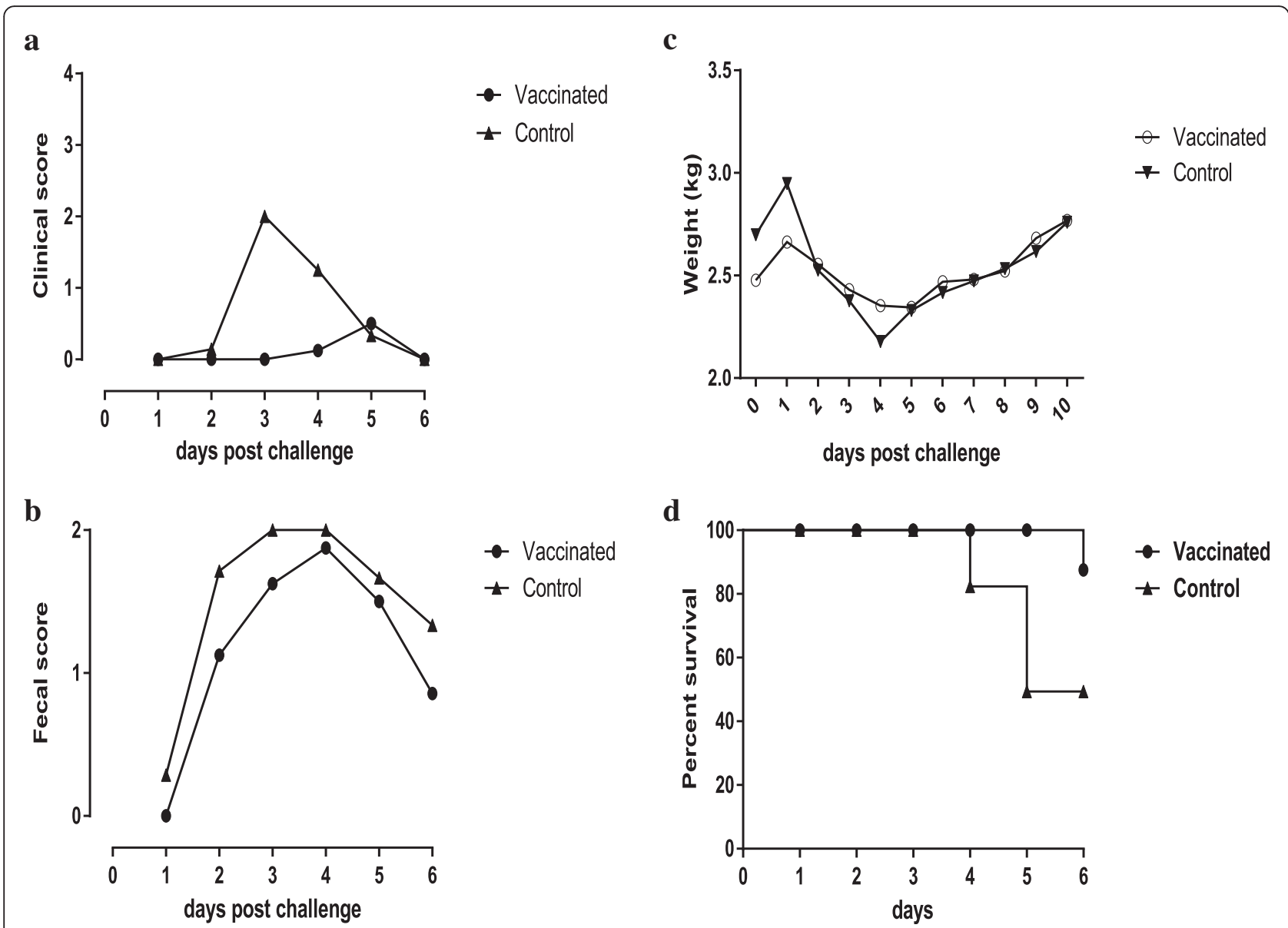

Fig. 8 Protective efficacy of the vaccine. Litters from a vaccinated $(n=8)$ and a control sow $(n=7)$ were orally challenged with virulent PEDV at 4 days of age. Clinical (a), fecal scores (b), average weight (c) and survival of piglets (d) were evaluated

administered the IM vaccine with the same dose [26]. To deliver a recombinant protein orally, a live vector such as adenoviral vector might be used [27].

\section{Conclusions}

Amongst three eukaryotic expression systems, the highest production of S1 was achieved using a mammalian expression with the TPA signal sequence. This method of expression can be used to produce large amount of S1 for vaccine, diagnostic and research purposes.

A sow vaccinated three times with the $\mathrm{S} 1$ subunit vaccine had high IgG titres and high virus neutralization titres in the serum as compared to the control sow. Also, high levels of IgG and IgA titers were found in the colostrum of the vaccinated sow. Furthermore, maternal transfer of antibody was demonstrated as only the serum of suckling piglets had high titres of S1 specific IgG and viral-neutralizing antibody. There was higher survival rate among of piglets of vaccinated sow (87.5\%) than in the piglets of control sow (42.9\%) after oral PEDV challenge $(P=0.0002)$. These survival rates suggest the potential of the S1 protein subunit vaccine in preventing piglet mortality. The vaccinated sow's piglets exhibited lower clinical signs of disease except diarrhea throughout the six day post-challenge period than control piglets. Surprisingly, there was no difference in average weight and PEDV shedding among two groups of piglets. These observations suggest the inability of the S1 subunit vaccine in our experiment to control all PED clinical signs although more experiments involving a large number of sows are needed to confirm these findings.

\section{Methods}

\section{Cells and virus}

Human embryo kidney (HEK) 293 T (ATCC CRL-1573) and Vero 76 (ATCC CRL-1587) cells were cultured in Dulbecco's modified Eagle medium (DMEM) supplemented with $50 \mu \mathrm{g} / \mathrm{mL}$ gentamycin and $10 \%$ fetal bovine serum. PichiaPink Strain 1 (Invitrogen) was grown in YPD medium. Insect Sf9 cells (Gibco) were grown in Sf-900 serum-free medium (ThermoFisher Scientific). 
PEDV strain USA/Colorado/2013 (GeneBank accession no. KF272920) was provided by Diagnostic Virology Laboratory (NVLS, Ames, USA), and the virus was propagated on Vero 76 cells in DMEM containing $50 \mu \mathrm{g} / \mathrm{mL}$ gentamycin, $2 \mu \mathrm{g} / \mathrm{mL}$ TPCK-trypsin and $0.2 \%$ bovine serum albumin.

\section{Expression of recombinant S1 protein in yeast cells}

Yeast codon optimized PEDV S1 gene (coding animo acids $1-734$ ) with the 3 '-end polyhistidine coding sequence was synthesized by GenScript. Synthetic S1 gene was digested with restriction enzymes $M l y \mathrm{I}-K p n \mathrm{I}$ and cloned into the pPink $\alpha-H C$ vector (ThermoFisher Scientific) into StuI-KpnI sites. Expression of the recombinant protein was performed as recommended by manufacturers (ThermoFisher Scientific). Briefly, PichiaPink strain $1\left(\right.$ ade $\left.2^{-}\right)$was electroporated with pPink $\alpha$-S1 linearized plasmid DNA and plated on PAD (Pichia Adenine Dropout) agar plates for selecting transformants. After incubation at $30{ }^{\circ} \mathrm{C}$ for 5 days, white colonies were screened for expression of S1. Recombinant yeast cells were grown in buffered glycerol-complex medium (BMGY) and induction was performed in buffered methanol-complex medium (BMMY) with $0.5 \%$ methanol. To prevent non-specific cleavage of S1 protein by yeast cell proteases, aprotinin and phenylmethylsulfonyl fluoride (PMSF) were added during the induction phase at $1 \mu \mathrm{g} / \mathrm{mL}$ each. Yeast cells were grown at $30{ }^{\circ} \mathrm{C}$ for 4 days and centrifuged at $3000 \mathrm{~g}$ for $5 \mathrm{~min}$ at room temperature and supernatant was collected for protein purification.

\section{Expression of recombinant $\mathrm{S} 1$ protein in insect cells}

S1 coding region of PEDV genome was PCR amplified using primers (5'-TCCGATGAATTCGCCACCATGAA GTCACTCACCTATTTTTGG-3' and 5'- CTAGATCTC GAGTCAGTGGTGATGATGGTGGTGGAAGCCAGG GAGTTCGCGG-3'). The resulting PCR product was cloned into the XhoI, EcoRI sites of pFastBac vector (ThermoFisher Scientific). Cellfectin ${ }^{\circ}$ II Reagent (ThermoFisher Scientific) was used for transfecting Sf9 cells in Grace's insect cell culture medium (ThermoFisher Scientific) for generating recombinant baculovirus. All the steps of generating the recombinant baculovirus full length genome, screening, rescue of recombinant baculovirus and large scale production of S1 protein in Sf9 cells was performed according to manufacturer's recommendations for Bac-to-Bac Baculovirus Expression System (ThermoFisher Scientific). Briefly, the Sf9 cells were grown at $30{ }^{\circ} \mathrm{C}$ for $24 \mathrm{~h}$ in the Sf-900 II SFM (ThermoFisher Scientific) at an orbital shaker incubator in two $1 \mathrm{~L}$ flasks (500 $\mathrm{mL}$ culture volume). Sf9 cells were infected with the recombinant baculovirus at an MOI of 1 and kept for 7 days in the incubator for secretion of the recombinant S1 protein. The supernatant was collected by centrifuging the insect cells at $500 \mathrm{~g}$ for $5 \mathrm{~min}$ at room temperature.

\section{Expression of recombinant $\mathrm{S} 1$ protein in mammalian cells} The S1 ORF plus C-terminal his ${ }_{10}$ tag (codon optimized for mammalian expression) together with a proceeding Kozak sequence, was cloned downstream of a human CMV promoter plus intron, contained within an inhouse episomal vector; elements downstream of the S1 ORF included a woodchuck hepatitis post-transcriptional regulatory element and bovine growth hormone polyadenylation site (DNA sequence of constructs are available upon request). Constructs were transfected into HEK293T cells using Turbofect (ThermoFisher Scientific) according to the manufacturer's instructions. Cells stably maintaining the episomal constructs were selected by puromycin. For protein production, stably transfected cells were grown in SFM4HEK293 medium (ThermoFisher Scientific) with shaking at $37^{\circ} \mathrm{C}$ and $5 \% \mathrm{CO}_{2}$. Supernatant was harvested and processed for purification.

\section{Purification of recombinant S1 protein}

Purifications of supernatants from the recombinant yeast cells and recombinant baculovirus infected cells were performed in the same way. First, equal volume of wash buffer was added to the samples $(50 \mathrm{mM}$ sodium phosphate, $0.3 \mathrm{M}$ sodium chloride, $10 \mathrm{mM}$ imidazole $\mathrm{pH} 8.0$ ) and then $\mathrm{pH}$ was adjusted to 8.0. Next, the samples were passed through $0.2 \mu \mathrm{m}$ filters to remove cellular debris and applyed to the HisSelect Ni Affinity (Sigma Aldrich) column. The column was washed with three sample volumes of wash buffer, followed by protein elution in one sample volume of elution buffer $(50 \mathrm{mM}$ sodium phosphate, $0.3 \mathrm{M}$ sodium chloride, $250 \mathrm{mM}$ imidazole $\mathrm{pH}$ 8.0). The eluate was concentrated using the Amicon Ultra-15 (EMD Millipore) filters and protein concentration was determined by a Bradford assay, and the protein quality was analysed by Western blotting.

Supernatants from HEK293T cultures were concentrated 5-fold by tangential flow and protein was purified using His60 Ni Superflow (Clontech) in accordance with the manufacturer's instructions. Purified protein was dialysed against PBS and quantified using a Bradford assay.

\section{Glycan analysis}

PNGase $\mathrm{F}$ and O-Glycosidase was purchased from NEB. Briefly, $2 \mu \mathrm{g}$ of purified S1 protein was added with $1 \mu \mathrm{L}$ of 10X glycoprotein denaturing buffer (NEB) in total of $10 \mu \mathrm{L}$ of reaction volume and denatured at $95{ }^{\circ} \mathrm{C}$ for $5 \mathrm{~min}$. Then, the mixture was chilled on ice for $30 \mathrm{~s}$ followed by centrifugation for $10 \mathrm{~s}$ at $10,000 \mathrm{X}$ g. Reaction volume was increased to $20 \mu \mathrm{L}$ by adding $2 \mu \mathrm{L} 10 \mathrm{X}$ GlycoBuffer (NEB), $2 \mu \mathrm{L} 10 \% \mathrm{NP} 40$, water and $1 \mu \mathrm{L}$ of 
enzyme PNGase F or $2 \mu \mathrm{L}$ of O-glycosidase and incubated at $37{ }^{\circ} \mathrm{C}$ for $1 \mathrm{~h}$. The extent of glycosylation was analyzed by mobility shift on SDS-PAGE followed by Western blotting.

\section{Western blotting}

Protein samples were heated in the SDS sample loading buffer $(0.375 \mathrm{M}$ Tris pH 6.8, 12 \% SDS, 60 \% glycerol, $0.6 \mathrm{M}$ DTT, $0.06 \%$ bromophenol blue) at $95{ }^{\circ} \mathrm{C}$ for $5 \mathrm{~min}$. Samples were separated by electrophoresis in $10 \%$ SDS-PAGE followed by transfer of proteins onto nitrocellulose membrane in Towbin's buffer $(0.025 \mathrm{M}$ Tris, $0.192 \mathrm{M}$ glycine, $20 \%$ methanol) at $4{ }^{\circ} \mathrm{C}$ for $1 \mathrm{~h}$ at $100 \mathrm{~V}$. The membrane was blocked with Blocker Blotto (Thermo Scientific) at room temperature for $1 \mathrm{~h}$. The membrane was incubated in anti-His rabbit antibody (1:2000) in Tris-buffered saline $(0.1 \mathrm{M}$ Tris, $0.9 \% \mathrm{NaCl})$ added with $0.1 \%$ Tween- 20 and $1 \%$ skim milk at $4{ }^{\circ} \mathrm{C}$ on orbital shaker overnight. Membrane was washed three times in TBST, and alkaline phosphate goat antirabbit IgG antibody (1:5000) was added and incubated at room temperature for $1 \mathrm{~h}$ on orbital shaker. Unbound antibody was washed by three TBST washes and bands were visualized using an AP Conjugate Substrate Kit (BioRad).

\section{Pig immunization}

All the animal experiments were performed in the animal containment level 3 laboratories of VIDO-InterVac. All pigs were maintained and euthanized as per the protocol, approved by the University of Saskatchewan's Animal Research Ethics Board and adhered to the Canadian Council on Animal Care guidelines for humane animal use. Two commercial crossbred pregnant sows were used in this study. The sows were vaccinated intramuscularly on both sides of neck with either purified S1 protein $(400 \mu \mathrm{g}$ per dose) or saline mixed with TriAdj adjuvant [28] three times, 14 days apart (days 0, 14 and 28). Blood samples were collected for serum on days 0 , 11, 28 and 35. Farrowing was induced at 16 days after the last vaccination. Colostrum samples were collected on the day of farrowing. Piglets were allowed to suckle their dams, and on the 4th day of their life they were orally challenged with live PEDV $\left(3 \times 10^{2} \mathrm{TCID}_{50}\right.$ per piglet). Clinical signs, diarrhea, death and weight of challenged piglets were monitored daily throughout the study. Blood samples of all the piglets were collected on the day of challenge for analysis. Rectal swabs were collected from each of the piglets daily for analyzing the presence of viral RNA by qRT-PCR.

\section{Elisa}

Immulon 2 HB 96-well plates (ThermoFisher Scientific) were coated overnight with $0.1 \mathrm{ml} /$ well of $0.5 \mu \mathrm{g} / \mathrm{ml}$ purified recombinant $\mathrm{S} 1$ protein. The plates were washed five times in phosphate buffer saline (PBS) containing $0.05 \%$ Tween 20 . Sera serially diluted in assay diluent buffer (0.1 M PBST, $0.05 \%$ Tween-20, $1 \%$ fish gelatin) were added to respective wells. After a $2 \mathrm{~h}$ incubation, the plates were washed, and 1/3000 diluted HRPconjugated anti-pig IgG antibodies were added. After $1 \mathrm{~h}$ incubation, the plates were washed, and developed with 1-Step Ultra TMB-ELISA substrate solution (ThermoFisher Scientific). The reaction was stopped by adding $30 \mu \mathrm{L}$ of $2 \mathrm{M}$ sulphuric acid to each well, and optical density values were measured at $450 \mathrm{~nm}$ using an ELISA plate reader.

A colostrum sample $(1 \mathrm{~mL})$ collected on the day of farrowing was treated with $30 \mu \mathrm{L}$ rennet $(5 \mathrm{mg} / \mathrm{mL}$, SigmaAldrich). The colostrum was then incubated at $37^{\circ} \mathrm{C}$ for $1 \mathrm{~h}$. Once solidified, the whey was separated by centrifugation at $6000 \mathrm{x}$ g for $20 \mathrm{~min}$. Whey samples were diluted in PBS containing $0.05 \%$ Tween 20 and $1 \%$ casein and applied at four-fold dilution on ELISA plate coated with the purified S1 protein. Plate was incubated at room temperature for $2 \mathrm{~h}$. The plates were washed six times with water between each step. Mouse anti-pig IgA (AbD Serotec) was applied to the plate at 1:300 dilution and incubated for $1 \mathrm{~h}$. Donkey anti-mouse HRP conjugate (Jackson Immunoresearch) was applied at 1:5000 dilution and incubated for $1 \mathrm{~h}$, and 1-Step Ultra TMB-ELISA substrate solution (ThermoFisher Scientific) was applied to the plates to develop the reaction. Then, the reaction was stopped and red as described above.

\section{Serum neutralization}

The presence of PEDV-specific neutralizing antibodies in serum of sows and piglets was determined using a serum neutralizing ( $\mathrm{SN}$ ) test. Briefly, serum samples were diluted 2 -fold and mixed with an equal volume of 200 TCID $_{50}$ of PEDV in each well. After a $1 \mathrm{~h}$ incubation at $37{ }^{\circ} \mathrm{C}$, $100 \mu \mathrm{L}$ of virus-serum mix was added to 96-well microtiter plate with a confluent monolayer of Vero 76 cells. The cells were incubated for $3 \mathrm{~h}$ at $37{ }^{\circ} \mathrm{C}$ in $5 \% \mathrm{CO}_{2}$ and unbound virus particles were removed with two washes of DMEM. Then, $100 \mu \mathrm{L}$ DMEM supplemented with trypsin $(2 \mu \mathrm{g} / \mathrm{mL})$ was added to the cells and incubated for $1 \mathrm{~h}$ at $37{ }^{\circ} \mathrm{C}$ in $5 \% \mathrm{CO}_{2}$. Thereafter, $100 \mu \mathrm{L}$ DMEM containing trypsin $(2 \mu \mathrm{g} / \mathrm{mL})$ and albumin $(0.2 \%)$ were added to each well and the cells were incubated for the period of 7 days at $37^{\circ} \mathrm{C}$ in $5 \% \mathrm{CO}_{2}$. The virus neutralizing antibody titers were expressed as the reciprocal of the highest serum dilution that showed no CPE in the cells. PEDV positive and negative control sera were also included in the tests.

\section{Viral RNA isolation and qRT-PCR}

Piglet fecal swabs were collected in $0.5 \mathrm{ml}$ DMEM on every day post-infection and stored at $-80{ }^{\circ} \mathrm{C}$. RNeasy 
Plus Kit (Qiagen) was used for RNA isolation from the faecal swab samples. qRT-PCR was conducted in two steps: cDNA synthesis and PCR reactions. cDNA synthesis was performed with $1 \mu \mathrm{L}(50 \mathrm{ng} / \mu \mathrm{L})$ random hexamers, $1 \mu \mathrm{L}$ of $10 \mathrm{mM}$ dNTPs, and RNA in $13 \mu \mathrm{L}$ volume and heated at $65{ }^{\circ} \mathrm{C}$ for $5 \mathrm{~min}$ and chilled on ice followed by addition of $4 \mu \mathrm{L}$ of $5 \mathrm{X}$ First-strand buffer, $1 \mu \mathrm{L}$ of $0.1 \mathrm{M}$ DTT and $1 \mu \mathrm{L}$ of RNaseOUT (ThermoFisher Scientific) and $1 \mu \mathrm{L}$ of SuperScript III enzyme (ThermoFisher Scientific) in final volume of $20 \mu \mathrm{L}$. The reaction conditions include $25{ }^{\circ} \mathrm{C}$ for $5 \mathrm{~min}, 50{ }^{\circ} \mathrm{C}$ for $60 \mathrm{~min}$ and $70{ }^{\circ} \mathrm{C}$ for $15 \mathrm{~min}$. The PCR reaction was performed in a total volume of $20 \mu \mathrm{L}$ with $2 \mu \mathrm{l} \mathrm{cDNA}$ using Power SYBR green Master Mix (Qiagen); primers (5'GCAACAACAGGTCCAGATCTC-3' and 5'-CTCCACG ACCCTGGTTATTTC-3') were present at $0.5 \mu \mathrm{M}$. PCR cycling conditions were: $95{ }^{\circ} \mathrm{C}$ for $10 \mathrm{~min}$ and 41 cycles of $95^{\circ} \mathrm{C}$ for $15 \mathrm{~s}, 60^{\circ} \mathrm{C}$ for $1 \mathrm{~min}$.

\section{Statistical analysis}

All data were analyzed using the GraphPad Prism (Version 6) software. Differences between two groups were assessed using unpaired two-tailed $t$-test. Differences were considered significant if $P<0.05$. Survival curves were created using the product limit method of Kaplan and Meier, and comparison of the curves was done using the logrank test.

\section{Competing interests}

The authors declare that they have no competing interests.

\section{Authors' contributions}

NM carried out the most of the experiments, participated in the data analysis and drafted the manuscript. RB helped to express and purify recombinant proteins. JvdH carried out the virus neutralization assay. NB performed qRT-PCR. BA participated in the data analysis. VG participated in the design of the study. AZ conceived of the study, and participated in its design and coordination and helped to draft the manuscript. All authors read and approved the final manuscript.

\section{Acknowledgements}

We thank Shirley Hauta, Yurij Popowych, Elaine van Moorlehem and Yan Zhou for help in the virus production. We thank Stew Walker, Colette Wheeler and Don Wilson for help in establishing a PEDV challenge model and providing veterinary expertize. This paper was published with the permission of the Director of VIDO-InterVac, journal series no. 770.

Received: 4 January 2016 Accepted: 22 March 2016

Published online: 01 April 2016

\section{References}

1. Pijpers A, van Nieuwstadt AP, Terpstra C, Verheijden JH. Porcine epidemic diarrhoea virus as a cause of persistent diarrhoea in a herd of breeding and finishing pigs. Vet Rec. 1993;132:129-31.

2. ICTV. Virus taxonomy: classification and nomenclature of viruses: Ninth Report of the International Committee on Taxonomy of Viruses. San Diego: Elsevier Academic Press; 2012.

3. Lee N, Hui D, Wu A, Chan P, Cameron P, Joynt GM, Ahuja A, Yung MY, Leung CB, To KF. A major outbreak of severe acute respiratory syndrome in Hong Kong. N Engl J Med. 2003;348:1986-94.

4. Zaki AM, van Boheemen S, Bestebroer TM, Osterhaus AD, Fouchier RA. Isolation of a novel coronavirus from a man with pneumonia in Saudi Arabia. N Engl J Med. 2012;367:1814-20.
5. Saif $L$. Animal coronaviruses: what can they teach us about the severe acute respiratory syndrome? Rev Sci Tech. 2004;23:643-60.

6. Pensaert MB, de Bouck P. A new coronavirus-like particle associated with diarrhea in swine. Arch Virol. 1978;58:243-7.

7. Li W, Li H, Liu Y, Pan Y, Deng F, Song Y, Tang X, He Q. New variants of porcine epidemic diarrhea virus, China, 2011. Emerg Infect Dis. 2012;18: 1350-3.

8. Sun RQ, Cai RJ, Chen YQ, Liang PS, Chen DK, Song CX. Outbreak of porcine epidemic diarrhea in suckling piglets, China. Emerg Infect Dis. 2012;18:161-3.

9. Chen Q, Li G, Stasko J, Thomas JT, Stensland WR, Pillatzki AE, Gauger PC, Schwartz KJ, Madson D, Yoon KJ. Isolation and characterization of porcine epidemic diarrhea viruses associated with the 2013 disease outbreak among swine in the United States. J Clin Microbiol. 2014;52:234-43.

10. Ojkic D, Hazlett M, Fairles J, Marom A, Slavic D, Maxie G, Alexandersen S, Pasick J, Alsop J, Burlatschenko S. The first case of porcine epidemic diarrhea in Canada. Can Vet J. 2015;56:149-52.

11. Lin CN, Chung WB, Chang SW, Wen CC, Liu H, Chien CH, Chiou MT. US-like strain of porcine epidemic diarrhea virus outbreaks in Taiwan, 2013-2014. J Vet Med Sci. 2014;76:1297-9.

12. Stadler J, Zoels S, Fux R, Hanke D, Pohlmann A, Blome S, Weissenbock H, Weissenbacher-Lang C, Ritzmann M, Ladinig A. Emergence of porcine epidemic diarrhea virus in southern Germany. BMC Vet Res. 2015;11:142.

13. Mesquita JR, Hakze Van Der Honing R, Almeida A, Lourenco M, Van Der Poel WH, Nascimento MS. Outbreak of porcine epidemic diarrhea virus in portugal, 2015. Transbound Emerg Dis. 2015;62:586-8.

14. Jung K, Saif LJ. Porcine epidemic diarrhea virus infection: Etiology, epidemiology, pathogenesis and immunoprophylaxis. Vet J. 2015;204:134-43.

15. Song D, Moon H, Kang B. Porcine epidemic diarrhea: a review of current epidemiology and available vaccines. Clin Exp Vaccine Res. 2015;4:166-76.

16. Kocherhans R, Bridgen A, Ackermann M, Tobler K. Completion of the porcine epidemic diarrhoea coronavirus (PEDV) genome sequence. Virus Genes. 2001;23:137-44.

17. Gallagher TM, Buchmeier MJ. Coronavirus spike proteins in viral entry and pathogenesis. Virology. 2001;279:371-4.

18. Li BX, Ge JW, Li YJ. Porcine aminopeptidase $\mathrm{N}$ is a functional receptor for the PEDV coronavirus. Virology. 2007;365:166-72.

19. Wicht O, Li W, Willems L, Meuleman TJ, Wubbolts RW, van Kuppeveld FJ, Rottier PJ, Bosch BJ. Proteolytic activation of the porcine epidemic diarrhea coronavirus spike fusion protein by trypsin in cell culture. J Virol. 2014;88: 7952-61.

20. Hofmann M, Wyler R. Propagation of the virus of porcine epidemic diarrhea in cell culture. J Clin Microbiol. 1988;26:2235-9.

21. Cruz DJ, Kim CJ, Shin HJ. The GPRLQPY motif located at the carboxyterminal of the spike protein induces antibodies that neutralize Porcine epidemic diarrhea virus. Virus Res. 2008;132:192-6.

22. Sun D, Feng L, Shi H, Chen J, Cui X, Chen H, Liu S, Tong Y, Wang Y, Tong G. Identification of two novel $B$ cell epitopes on porcine epidemic diarrhea virus spike protein. Vet Microbiol. 2008;131:73-81.

23. Chang SH, Bae JL, Kang TJ, Kim J, Chung GH, Lim CW, Laude H, Yang MS, Jang YS. Identification of the epitope region capable of inducing neutralizing antibodies against the porcine epidemic diarrhea virus. Mol Cells. 2002;14:295-9.

24. Oh J, Lee KW, Choi HW, Lee C. Immunogenicity and protective efficacy of recombinant S1 domain of the porcine epidemic diarrhea virus spike protein. Arch Virol. 2014;159:2977-87.

25. Salmon H, Berri M, Gerdts V, Meurens F. Humoral and cellular factors of maternal immunity in swine. Dev Comp Immunol. 2009;33:384-93.

26. Song DS, Oh JS, Kang BK, Yang JS, Moon HJ, Yoo HS, Jang YS, Park BK. Oral efficacy of Vero cell attenuated porcine epidemic diarrhea virus DR13 strain. Res Vet Sci. 2007;82:134-40.

27. Ferreira TB, Alves PM, Aunins JG, Carrondo MJ. Use of adenoviral vectors as veterinary vaccines. Gene Ther. 2005;12 Suppl 1:S73-83.

28. Garg R, Latimer L, Simko E, Gerdts V, Potter A, van den Hurk S. Induction of mucosal immunity and protection by intranasal immunization with a respiratory syncytial virus subunit vaccine formulation. J Gen Virol. 2014;95:301-6. 